\title{
The phytological future of prostate cancer staging: PSMA-PET and the dandelion theory
}

\author{
Niranjan J Sathianathen', Nicolas Geurts', Rajesh Nair', Nathan \\ Lawrentschuk $^{1,2}$, Declan G Murphy ${ }^{1,3}$ \& Alastair D Lamb ${ }^{* 1,4,5}$
}

\begin{abstract}
PSMA-PET is changing how we stage prostate cancer, both in the primary setting and with relapse after treatment. It allows us to identify lesions in the bones and lymph nodes that were not previously visible on conventional imaging with bone scan and CT/MRI. In this Special Report we review the 'state of the art' for PSMA imaging and discuss the implications for treatment decisions in prostate cancer. We liken early high risk or metastatic prostate to a common phytological problem: the dandelion. In this analogous situation, we consider the additional evidence needed for us to start plucking out the original dandelion and for us to focus attention on killing the seeded weeds that are identifiable elsewhere in the lawn.
\end{abstract}

First draft submitted: 15 April 2017; Accepted for publication: 19 May 2017; Published online: 1 August 2017

Anybody who has spent more than a few hours cutting or rolling a newly laid lawn will be familiar with the blight of dandelions. Catch one before it is seeded and your lawn stands a chance of retaining its pristine lines and surface (Figure 1). But come out one windy evening to find a freshly seeded flower head and all is lost. Even if you pluck it out you can be certain that the tiny seed pods have been carried to all four corners of your lawn and, given a month, will make their presence known. So much for gardening, but cancer surgery is often similar.

One of the core principles of radical surgery is accurate staging in the preoperative setting. For most cancers, it is essential to confirm that the tumor is localized - that is, has not yet seeded - such that radical resection can reasonably be expected to achieve a cure [1]. In prostate cancer, various imaging methods have been employed to provide this information, with European Guidelines currently recommending MRI for local staging and CT/bone scan for exclusion of distant metastases for those with intermediate or high-risk disease [2]. However, it is generally accepted that CT/bone scan has low sensitivity and specificity for detection of metastatic disease, with small foci often missed, or conversely nonspecific lesions being detected such as degenerative bone disease or reactive inflammatory changes in the lymph nodes. This being the case, many men with 'localized disease' deemed to be candidates for radical surgery over the past few decades are likely, in fact, to have had small volume or micrometastatic disease. Although it is hard to tease out which subset of men undergoing surgery are in this group, they form part of a cohort in whom we have seen excellent oncological

'Department of Genitourinary Oncology \& Division of Cancer Surgery, Peter MacCallum Cancer Centre, The University of Melbourne, Victoria, Australia

2Department of Surgery, Austin Health, The University of Melbourne, Victoria, Australia

${ }^{3}$ Australian Prostate Cancer Research Centre, Epworth Healthcare, Richmond, Australia

${ }^{4}$ Academic Urology Group, University of Cambridge, Addenbrooke's Hospital, Cambridge, UK

${ }^{5}$ Nuffield Department of Surgical Sciences, University of Oxford, UK

*Author for correspondence: alastair.lamb@nds.ox.ac.uk

\section{KEYWORDS}

- metastasis $\bullet$ PET $\bullet$ prostate cancer $\bullet$ PSMA • staging 


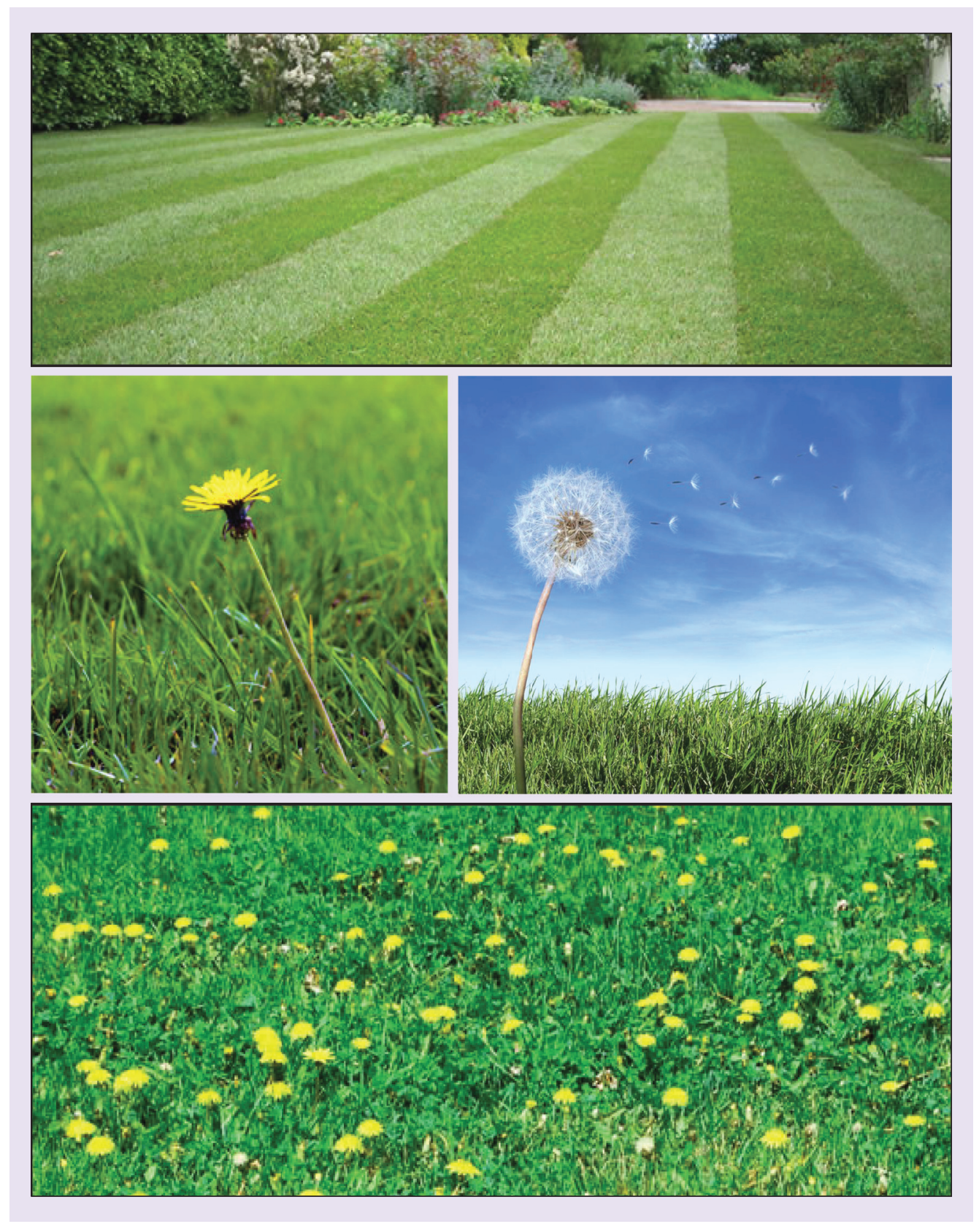

Figure 1. Dandelions proliferate in a garden left untended.

outcomes with 10-year disease-specific mortality of around $1 \%$ [3].

In this article, we highlight the way in which prostate-specific membrane antigen (PSMA) scanning has delivered a whole new level of accuracy in detection of 'seeded' prostate cancer, with the potential to dramatically change the way in which we select men for radical surgery, but also forcing us to re-interpret the way in which we have performed prostate cancer surgery to the present day.
What is PSMA?

PSMA is a transmembrane protein that generally has increased expression in prostate cancer cells relative to normal cells. This is the case both locally and in distant metastatic lesions, making it an attractive target for molecular imaging [4]. In addition, the degree of PSMA expression has been demonstrated to correlate with tumor aggressiveness [5]. Initial research on PSMA focused on the use of Indium-111 capromab pendetide (Prostascint ${ }^{\circledR}$, Englewood, 

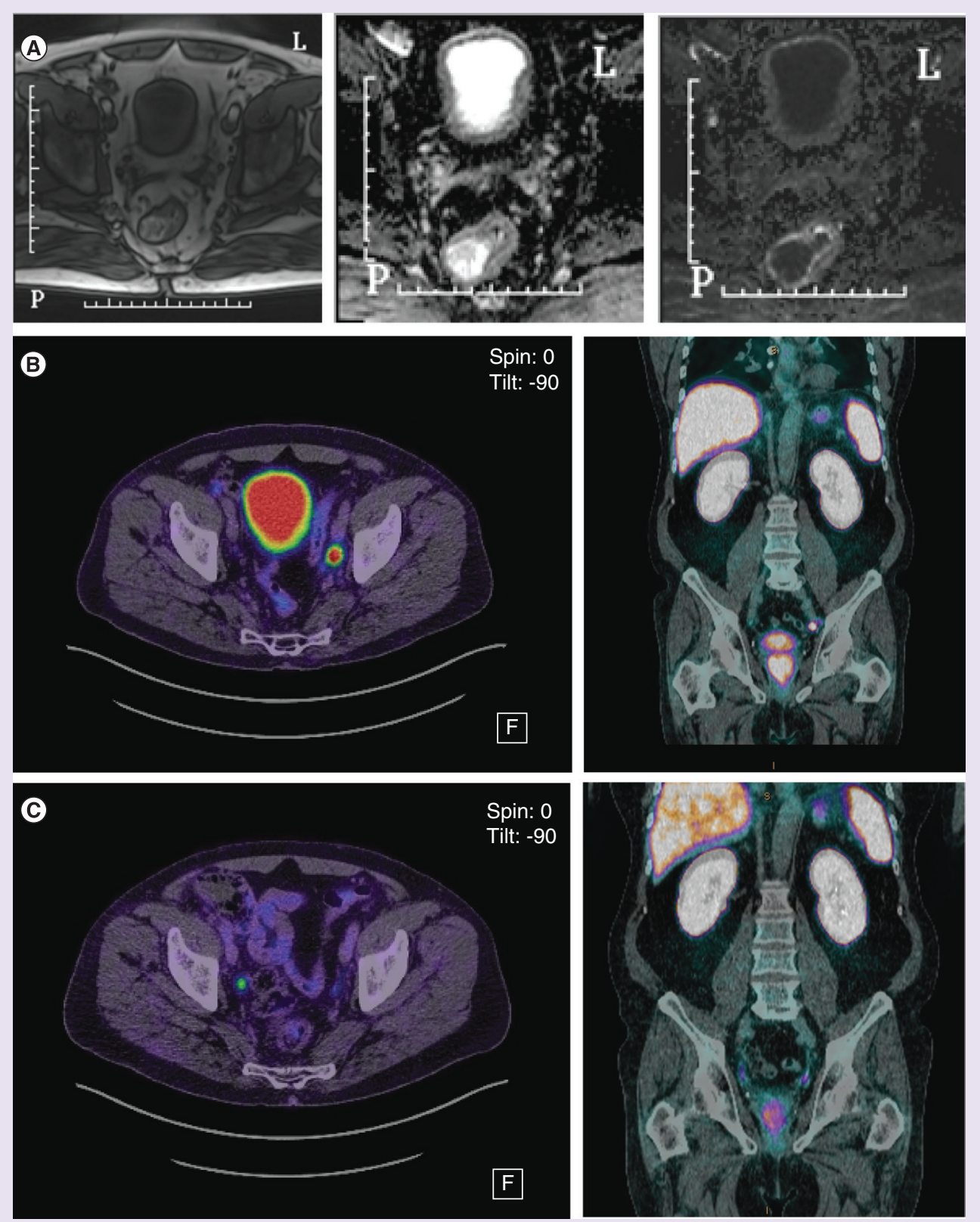

Figure 2. Prostate-specific membrane antigen-positron emission tomography enhances detection of nodal disease. (A) Conventional cross-sectional staging MRI scan showing no obvious nodal disease in a patient with Gleason Grade Group 3 prostate cancer and a prostate specific antigen of 85 deciding whether to undergo surgery. Despite the suspiciously high prostate spedific antigen his conventional staging did not reveal any disease. (B) prostate specific memrane antigenPET imaging, in contrast to traditional staging, revealed an obvious node in the left obturator fossa clearly seen on both axial and coronal planes. (C) The patient received 6 months of hormone therapy preoperatively and repeat PSMA scanning revealed almost complete resolution of the prostate spefic menbrane antigen avidity in the left obturator area on axial and coronal imaging. Urinary tract and liver show incidental avidity due to tracer excretion. 
CO, USA) and single-photon emission CT with limited success [6], however, more recent research has delivered promising results in exploring the utility of small-molecule PSMA ligands labeled with radioligand tracers in positron emission tomography (PET) scanning [7]. Of these, ${ }^{68} \mathrm{Ga}$-labeled PSMA inhibitor Glu-NH-CONH-Lys (Ahx)-HBED-CC has been the most widely studied with initial studies exhibiting its potential to revolutionize the prostate cancer imaging landscape. Other tracers with promising initial results include ${ }^{18} \mathrm{~F}$-Fluoride-DCFPyLPSMA that seem to be safe [8] and with high affinity [9].

\section{Superior detection of nodal metastases} In terms of nodal metastases, ${ }^{68} \mathrm{Ga}$-PSMA PET/ CT has been shown to be better than conventional imaging modalities (Figure 2). The inadequacy of CT in detecting nodal disease from prostate cancer was highlighted by Hovels et al. in their meta-analysis when they reported a pooled sensitivity and specificity of 42 and $82 \%$, respectively, with MRI not performing much better [10]. In contrast, a German study utilizing PSMA PET/CT reported a sensitivity and specificity of 66 and $99 \%$, respectively [11]. These PSMA results were validated by van Leeuwen et al. who published similar rates [12]. The utility of PSMA is at least equal, if not greater, in the secondary staging setting as in primary staging. Rauscher et al. reported $77.9 \%$ detection rate in histologically proven nodal disease compared with $26.9 \%$ when using conventional cross-sectional imaging [13]. Additionally, the mean size of positive nodes that were able to be detected by PSMA PET was $8.3 \mathrm{~mm}$ compared with $13.0 \mathrm{~mm}$ using CT or MRI, thus highlighting the superiority of PSMA scanning. The high quality of these studies should be emphasized as they were able to directly compare radiological findings to histological diagnosis. Furthermore, the once hyped use of choline PET tracers has also been surpassed by the diagnostic yield of ${ }^{68} \mathrm{Ga}$-PSMA. PSMA imaging detected recurrence in just under half of men who had previously negative ${ }^{18} \mathrm{~F}$-Choline-PET/CT [14].

Superior detection of bone metastases PSMA has also markedly enhanced the ability to visualize osseous metastases even when not visible with Technietium-99m-methylene disphonate (Tc-MDP) bone scanning or ${ }^{18} \mathrm{~F}$-Fluoride PET CT (Figure 3). The majority of clinical guidelines recommend the use of bone scintigraphy with Tc-MDP to assess for skeletal metastases, however, the overall specificity with this tracer is low because Technetium-99m-MDP is taken up in a range of nonmalignant processes. In a direct comparison with ${ }^{68} \mathrm{Ga}$-PSMA PET/ CT, Pyka et al. reported the sensitivity and specificity of detecting skeletal disease when using bone scintigraphy to be 86.7 and $60.8 \%$ compared with 98.7 and $88.2 \%$ when using PSMA [15]. Subgroup analysis further supported the superiority of PSMA in all studied clinical settings - primary staging, secondary staging and those with metastatic castrate-resistant prostate cancer. Despite the exquisite sensitivity and specificity of PSMA - a true example of bench-to-bedside translation in molecular science nonspecificity of PSMA seems to arise within certain nonmalignant processes such as Pagets disease [16], as well as certain other cancers including hepatocellular carcinoma, clear-cell kidney cancer and brain tumors [17].

PSMA PET scanning has demonstrated the capacity to reveal extraprostatic disease at an early stage. In a recent meta-analysis, Perera et al. reported a 48 and $70 \%{ }^{68} \mathrm{Ga}$-PSMA PET/CT predicted positivity with a PSA value 0.2 and $1.0 \mathrm{ng} / \mathrm{ml}$, respectively [18]. Similarly, a large German cohort with biochemical recurrence postprostatectomy supported these findings by demonstrating excellent detection rates over a range of PSA measurements (Table 1). In contrast, most clinical guidelines do not recommend performing conventional imaging in the secondary staging setting when the PSA is below 10 due to the low diagnostic yield [19].

Overall, the reported pooled sensitivity and specificity of PSMA imaging is 80 and $97 \%$, respectively [18].

\section{The potential for PSMA in oligometastatic} prostate cancer

This enhanced capability to detect metastatic lesions raises a number of clinical questions regarding treatment, especially when in the oligometastatic phase. The oligometastatic state is an intermediate stage of limited tumor spread before the onset of disseminated metastases [21]. The hypothesis, therefore, is that metastasis-directed therapy at this point should be beneficial in prostate cancer as in other malignancies such as colorectal tumors [22] However, there is conjecture regarding the exact definition of oligometastasis and thus where the line is drawn between oligometastases 


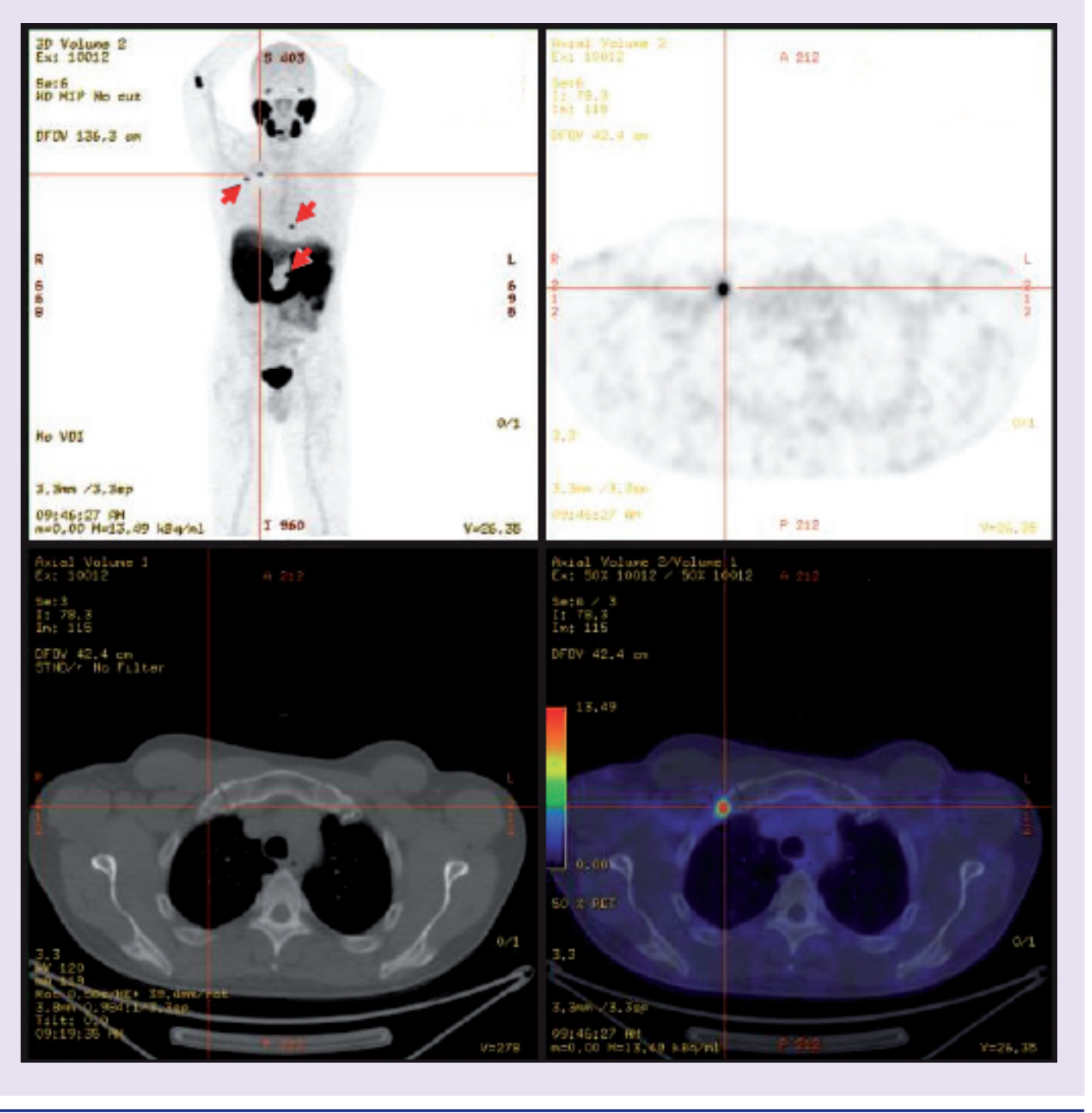

Figure 3. Prostate-specific membrane antigen-positron emission tomography enhances detection of skeletal metastases. Previous conventional bone scan imaging showed only a single metastasis in the right first rib in this man with Gleason Grade Group 5 prostate cancer who had previously undergone radical prostatectomy. However, prostate-specific membrane antigen scanning revealed four sites of skeletal disease including two further sites in the ribs and one in the lumbar spine (see red arrows on the maximum intensity projection - overview image top left). N.B. Nonspecific avidity is again seen in the urinary tract, liver and also in the salivary glands.

and disseminated disease. The majority of the published literature centers on quantity, with the number of lesions usually set at five. This is largely based on a study by Singh et al., who reported that patients with five or fewer metastatic sites had significantly better survival rates than those with more than five [23]. Nevertheless, the set limit of lesions varies between trials with one study even including ten lesions in their definition of oligometastatic disease. Some also place caveats on the site of metastasis [24]. Furthermore, there is marked heterogeneity between the imaging modalities used in different trials that inevitably impacts sensitivity of detection. Crucially, no publications to date have used ${ }^{68} \mathrm{Ga}$-PSMA PET/CT which, as outlined above, has been shown to be far superior to other modalities. This raises important questions. It is likely that patients who met the definition of oligometastatic disease in the literature would have had more widespread disease had PSMA scanning been used. Inevitably, the definition of oligometastasis will undergo further amendment in the future with clinical trials that use ${ }^{68} \mathrm{Ga}$-PSMA PET/CT providing clarity to this space.

Despite the ambiguity that surrounds the definition of oligometastatic disease; initial 
Table $1 .{ }^{68} \mathrm{Ga}$-Prostate-specific membrane antigen-positron emission tomography/CT detection rate based on PSA values.

\begin{tabular}{|c|c|c|}
\hline \multirow[t]{2}{*}{ PSA (ng/ml) } & \multicolumn{2}{|c|}{${ }^{68} \mathrm{Ga}-\mathrm{PSMA} \mathrm{PET} / \mathrm{CT}$ detection rate } \\
\hline & Perera et al. & Eiber et al. [20] \\
\hline $0.2-<0.5$ & \multirow[t]{2}{*}{$58 \%$} & $57.9 \%$ \\
\hline $0.5-<1$ & & $72.7 \%$ \\
\hline $1-<2$ & $76 \%$ & $93.0 \%$ \\
\hline$\geq 2$ & $95 \%$ & $96.8 \%$ \\
\hline
\end{tabular}

reports suggest that metastasis-directed therapy improves outcomes. In a cohort of men with biochemical recurrence after radical prostatectomy who had no visceral or bony metastases and only two or fewer nodal sites on ${ }^{11} \mathrm{C}$-choline PET, Suardi et al. reported a 59.3\% rate of biochemical response after salvage pelvic lymph node dissection at a median 81.1-month follow-up [25]. Impressively, the 8-year cancer-specific mortality-free survival and biochemical recurrence-free rates were 81 and $23 \%$, respectively. Similarly, a German group found that $46 \%$ experienced complete biochemical response after salvage pelvic lymph node dissection [26]. Just under half of this cohort received adjuvant radiotherapy following lymph node dissection but this subset of men did not exhibit an advantage in preventing clinical progression. Despite most patients eventually experiencing biochemical recurrence after salvage therapy, the latter study showed that $42.6 \%$ of the group remained symptomfree for 3 years. Similarly, salvage radiotherapy - especially stereotactic beam radiotherapy - has shown benefit with a 2 -year progression-free survival rate of $35 \%$ and a median 25 -month delay to androgen deprivation therapy [27]. It should be noted that there was considerable heterogeneity between the studies. The challenge, therefore, remains to identify precisely those who will benefit most from metastasis-directed therapy.

\section{Conclusion}

We have to ask ourselves two questions: first, is it worth removing the single dandelion in the middle of the lawn even if the flower head has seeded?
Of course, yes. Dandelions are perennials and will keep seeding the lawn if left alone. Second, is it worth removing the seeded dandelions we see distributed around the lawn a month later? Again, yes, for the same reason. But, if we transition from phytology to oncology, the answers are less clear cut. Logic would suggest the same argument. "PSMA scanning is giving us the information we need...to make accurate decisions in prostate cancer surgery." Time and well-designed clinical trials will give us the answers.

\section{Future perspective}

PSMA-PET imaging will increasingly become available to nuclear medicine departments. Accurate pre-operative staging with detection of bone and nodal metastases will inform treatment decisions. Decision making in relapse disease will be enhanced by acc urate assessment of loco-regional or disseminated recurrence. Further work is needed to determine the impact of this enhanced decision-making on survival.

\section{Financial \& competing interests disclosure} $D G$ Murphy and $N$ Lawrentschuk are both principal investigators for the ProPSMA study (HREC/16/ PMCC/130), which is funded by the Movember Foundation. The authors have no other relevant affliations or financial involvement with any organization or entity with a financial interest in or financial conflict with the subject matter or materials discussed in the manuscript apart from those disclosed.

No writing assistance was utilized in the production of this manuscript.

\section{EXECUTIVE SUMMARY}

- PSMA is a transmembrane protein exclusively expressed in prostate cells-PSMA-PET imaging permits detection of nodal and bone metastases with $90 \%$ accuracy.

- PSMA-PET imaging requires a synchrotron particle accelerator and is therefore limited to sites that have access to this facility. 


\section{References}

1 Halsted WS. I. The results of operations for the cure of cancer of the breast performed at the Johns Hopkins Hospital from June, 1889, to January, 1894. Ann. Surg. 20(5), 497-555 (1894).

2 Mottet N, Bellmunt J, Bolla M et al. EAU-ESTRO-SIOG guidelines on prostate cancer. Part 1: screening, diagnosis, and local treatment with curative intent. Eur. Urol. 71(4), 618-629 (2017).

3 Hamdy FC, Donovan JL, Lane JA et al. 10 -Year outcomes after monitoring, surgery, or radiotherapy for localized prostate cancer. N. Eng. J. Med. 375(15), 1415-1424 (2016).

4 Kiess AP, Banerjee SR, Mease RC et al. Prostate-specific membrane antigen as a target for cancer imaging and therapy. Q. J. Nucl. Med. Mol. Imaging 59(3), 241-268 (2015).

5 Ross JS, Sheehan CE, Fisher HA et al. Correlation of primary tumor prostatespecific membrane antigen expression with disease recurrence in prostate cancer. Clin. Can Res. 9(17), 6357-6362 (2003).

6 Taneja SS. ProstaScint ${ }^{\circledR}$ scan: contemporary use in clinical practice. Rev. Urol. 6(Suppl. 10), S19-S28 (2004).

7 Afshar-Oromieh A, Hetzheim H, Kratochwil $\mathrm{C}$ et al. The theranostic PSMA ligand PSMA-617 in the diagnosis of prostate cancer by PET/CT: biodistribution in humans, radiation dosimetry, and first evaluation of tumor lesions. J. Nucl. Med. 56(11), 1697-1705 (2015).

8 Dietlein M, Kobe C, Kuhnert G et al. Comparison of [(18)F]DCFPyL and [(68) Ga] Ga-PSMA-HBED-CC for PSMA-PET imaging in patients with relapsed prostate cancer. Mol. Imaging Biol. 17(4), 575-584 (2015).

9 Kelly J, Amor-Coarasa A, Nikolopoulou A et al. Synthesis and pre-clinical evaluation of a new class of high-affinity ${ }^{18} \mathrm{~F}$-labeled PSMA ligands for detection of prostate cancer by PET imaging. Eur. J. Nucl. Med. Mol. Imaging 44(4), 647-661 (2017).
10 Hovels AM, Heesakkers RA, Adang EM, Jager GJ, Strum S, Hoogeveen YL. The diagnostic accuracy of CT and MRI in the staging of pelvic lymph nodes in patients with prostate cancer: a meta-analysis. Clin. Radiol. 63(4), 387-395 (2008).

11 Maurer T, Gschwend JE, Rauscher I et al. Diagnostic efficacy of gallium-PSMA positron emission tomography compared to conventional imaging in lymph node staging of 130 consecutive patients with intermediate to high risk prostate cancer. J. Urol. 195(5), 1436-1443 (2016).

12 van Leeuwen PJ, Emmett L, Ho B et al. Prospective evaluation of 68Gallium-PSMA positron emission tomography/computerized tomography for preoperative lymph node staging in prostate cancer. BJU Int. 119(2), 209-215 (2017).

13 Rauscher I, Maurer T, Beer AJ et al. Value of 68Ga-PSMA HBED-CC PET for the assessment of lymph node metastases in prostate cancer patients with biochemical recurrence: comparison with histopathology after salvage lymphadenectomy. J. Nucl. Med. 57(11), 1713-1719 (2016).

14 Bluemel C, Krebs M, Polat B et al. ${ }^{68} \mathrm{Ga}-$ PSMA-PET/CT in patients with biochemical prostate cancer recurrence and negative ${ }^{18}$ F-Choline-PET/CT. Clin. Nucl. Med. 41(7), 515-521 (2016).

15 Pyka T, Okamoto S, Dahlbender M et al. Comparison of bone scintigraphy and 68Ga-PSMA PET for skeletal staging in prostate cancer. Eur. J. Nucl. Med. Mol. Imaging 43(12), 2114-2121 (2016).

16 Froehner M, Toma M, Zophel K, Novotny V, Laniado M, Wirth MP. PSMA-PET/ CT-positive Paget disease in a patient with newly diagnosed prostate cancer: imaging and bone biopsy findings. Case Rep. Urol. 2017, 1654231 (2017).

17 Rauscher I, Maurer T, Fendler WP, Sommer WH, Schwaiger M, Eiber M. (68)Ga-PSMA ligand PET/CT in patients with prostate cancer: how we review and report. Cancer Imaging 16(1), 14 (2016).
18 Perera M, Papa N, Christidis D et al. Sensitivity, specificity, and predictors of positive $68 \mathrm{Ga}$-prostate-specific membrane antigen positron emission tomography in advanced prostate cancer: a systematic review and meta-analysis. Eur. Urol. 70(6), 926-937 (2016).

19 Choueiri TK, Dreicer R, Paciorek A, Carroll PR, Konety B. A model that predicts the probability of positive imaging in prostate cancer cases with biochemical failure after initial definitive local therapy. J. Urol. 179(3), 906-910; discussion 910 (2008).

20 Eiber M, Maurer T, Souvatzoglou M et al. Evaluation of hybrid (6)(8) Ga-PSMA Ligand PET/CT in 248 patients with biochemical recurrence after radical prostatectomy. J. Nucl. Med. 56(5), 668-674 (2015).

21 Weichselbaum RR, Hellman S. Oligometastases revisited. Nat. Rev. Clin. Oncol. 8(6), 378-382 (2011).

22 Clark ME, Smith RR. Liver-directed therapies in metastatic colorectal cancer. J. Gastro. Oncol. 5(5), 374-387 (2014).

23 Singh D, Yi WS, Brasacchio RA et al. Is there a favorable subset of patients with prostate cancer who develop oligometastases? Intern. J. Radiat. Oncol. Biol. Phys. 58(1), 3-10 (2004).

24 Tosoian JJ, Gorin MA, Ross AE, Pienta KJ, Tran PT, Schaeffer EM. Oligometastatic prostate cancer: definitions, clinical outcomes, and treatment considerations. Nat. Rev. Urol. 14(1), 15-25 (2017).

25 Suardi N, Gandaglia G, Gallina A et al. Long-term outcomes of salvage lymph node dissection for clinically recurrent prostate cancer: results of a single-institution series with a minimum follow-up of 5 years. Eur. Urol. 67(2), 299-309 (2015).

26 Jilg CA, Rischke HC, Reske SN et al. Salvage lymph node dissection with adjuvant radiotherapy for nodal recurrence of prostate cancer. J. Urol. 188(6), 2190-2197 (2012).

27 Decaestecker K, De Meerleer G, Lambert $\mathrm{B}$ et al. Repeated stereotactic body radiotherapy for oligometastatic prostate cancer recurrence. Rad. Oncol. 9, 135 (2014). 